\title{
Effects of Intravitreal Injection of Hypoxia-Induced Umbilical Cord Mesenchymal Stem Cell Exosomes on Diabetic Retinopathy
}

Yan Fu ( $\nabla$ shzfuyan@126.com )

Baoding No 1 Central Hospital https://orcid.org/0000-0001-8876-992X

Zhao-Hui Gu

Baoding No 1 Central Hospital

Xiao-Ying Wen

Baoding No 1 Central Hospital

$\mathrm{Na}$ Yang

Baoding No 1 Central Hospital

\section{Research Article}

Keywords: exosomes, human umbilical cord mesenchymal stem cell, diabetic retinopathy, intravitreal injection

Posted Date: January 18th, 2022

DOI: https://doi.org/10.21203/rs.3.rs-994654/v2

License: (9) This work is licensed under a Creative Commons Attribution 4.0 International License. Read Full License 


\section{Abstract}

BACKGROUND: This study aims to investigate the effect of intravitreal injection of hypoxia-induced human umbilical cord mesenchymal stem cell exosomes (hypo-hucMSC-Exs) on retinal microvasculature in rats with diabetic retinopathy (DR) and to determine whether hypo-hucMSC-Exs exhibit greater effects on DR than human umbilical cord mesenchymal stem cell exosomes (hucMSC-Exs)

METHODS: Exosomes were isolated from normal hucMSCs culture media and hypoxic preconditioning culture media. transmission electron microscope, nanoparticle tracking analysis, and western blot were applied to identification of hucMSC-Exs. Streptozotocin-induced diabetic rats were used as a model for DR. Fundus fluorescein angiography (FFA) was conducted to evaluate retinal microvasculature changes in vivo 4 week, 8 weeks and 12 weeks following intravitreal injection.

RESULTS: The control group did not experience any significant change throughout the study. After STZ injection, all rats developed hyperglycemia. The development of retinal microvascular changes include tortuous vessels, massive microaneurysms and late leakage of fluorescein dye was visualized using FFA after induction of DR. This response was ameliorated in diabetic rats treated with hucMSC-Exs. To further investigate the effects of hypo-hucMSC-Exs we found that injection with hypo-hucMSC-Exs remarkably reduced the microvasculature lesions compared with the hucMSC-Exs group.

CONCLUSION: These findings suggest that intravitreal injection of hucMSC-Exs prevented diabetesinduced microvasculature lesions. hypo-hucMSC-Exs could enhance this effects and could be considered as a potential target for DR prevention and treatment.

\section{Background}

Diabetic retinopathy (DR) is a common microvascular complication of diabetes mellitus and the main cause of blindness in adults. Blindness caused by DR is preventable but irreversible and represents a huge economic burden on families and society. Microvascular dysfunction is a major characteristics of $\mathrm{DR}$, which is diagnosed on the basis microvascular lesions [1]. Laser treatment, anti-vascular endothelial growth factor therapy, and vitrectomy are commonly used to treat DR; however, not all patients respond well to these approaches.

In recent years, the study of exosomes has gradually attracted increasing attention, opening up potential new avenues for treatment of retinal diseases. Specifically, research has shown that exosomes are involved in the occurrence and progression of many retinal diseases including DR, and they have potential value for application in the diagnosis and targeted treatment of DR.

Mesenchymal stem cells (MSCs) are derived from a wide range of tissues, are easy to and culture in vitro, and secrete relatively large amounts of exosomes, making them the best source cells for basic and clinical studies in exosome research. Vitreous MSC injection can exert a protective effect on the retina in DR by secreting various neurotrophic factors [2]. Oxygen concentration has an important role in the 
processes of MSC proliferation, differentiation, and self-renewal [3] [4]. Under hypoxia, MSCs can exhibit enhanced biological function and improved therapeutic efficacy $[3,5]$. Furthermore, MSC-derived exosomes have great potential for clinical application. For example, MSCs grown in a medium with conditions similar to those in peripheral artery disease $\left(0 \%\right.$ fetal bovine serum [FBS], $1 \% \mathrm{O}_{2}$ ) generate exosomes containing numerous pro-angiogenic factors that could be beneficial to ischemic tissue [6]. Another study using a model of heart infarction showed that hypoxia-induced MSC-derived exosomes increased vascularization, reduced myocardial apoptosis rate, and elevated numbers of cardiac progenitor cells [7]. Hence, hypoxic preconditioning of MSCs can significantly enhance their biological function, thereby improving their curative effects $[8,9]$. Since exosome content is greatly affected by the microenvironment in which the host cells reside [10], exosome levels can be improved by hypoxic preconditioning of source of MSCs. Hypoxic preconditioning can enhance MSC-derived exosome function in models of myocardial infarction [11], spinal cord injury [12], and bone fracture [13]; however, it remains unclear whether exosomes from MSCs exposed to hypoxic conditions exert better therapeutic effects in DR. Since the hypoxic microenvironment is a prominent feature of DR, we questioned whether hypoxiainduced human umbilical cord mesenchymal stem cell exosomes (hypo-hucMSC-Exs) would ameliorate DR-induced retinal vascular injury.

In this study, we established a streptozotocin (STZ)-induced DR model in rats to test the hypothesis that exosomes from MSCs grown under hypoxia would have better therapeutic efficacy than exosomes from MSCs cultured under normoxic conditions. The DR model was used to determine whether intravitreal injection of hypo-hucMSC-Exs had better protective effects on the retina than that of hucMSC-Exs not exposed to hypoxic conditions.

\section{Materials And Methods \\ 2.1 Ethics statement}

All experiments involving animals were performed in accordance with the Animals for Scientific Procedures Act 1986 and the ARVO Statement for the Use of Animals in Ophthalmic and Vision Research, and the study protocols were approved by the Medicine Ethics Review Committee for Animal Experiments of our hospital.

\subsection{Culture, hypoxia preconditioning, and isolation of hucMSC-Exs}

HucMSCs were purchased from Tianjin Saier Biological Company, China. HucMSCs at passage 3-5, with strong proliferation capacity were used for further experiments. Cells were cultured in a normoxic incubator at $37^{\circ} \mathrm{C}, 5 \% \mathrm{CO}_{2}, 21 \% \mathrm{O}_{2}$, or in a hypoxic cell incubator at $1 \% \mathrm{O}_{2}, 5 \% \mathrm{CO}_{2}$, in exosome-depleted FBS-containing media (System Biosciences, Mountain View, CA, USA) for $48 \mathrm{~h}$. HucMSC culture medium was collected by centrifugation at $1,000 \times \mathrm{g}\left(30 \mathrm{~min}, 4^{\circ} \mathrm{C}\right)$. Then, the supernatant was sequentially centrifuged for $10 \mathrm{~min}$ at $300 \times \mathrm{g}, 20 \mathrm{~min}$ at 2,000 $\times \mathrm{g}$, and $30 \mathrm{~min}$ at 10,000 $\times \mathrm{g}$, and filtered through a 
$0.22-\mu \mathrm{m}$ filter. Exosomes were then precipitated by ultracentrifugation of the supernatant (70 min, $100,000 \times \mathrm{g})$, resuspended in phosphate-buffered saline (PBS) $(25 \mu \mathrm{LBS} / 1 \mathrm{~mL}$ culture medium), and stored at $-80^{\circ} \mathrm{C}$ for later use.

\subsection{Characterization of hucMSC-Exs}

HucMSC-Ex morphology was characterized using an FEI Tecnai G2 transmission electron microscope (TEM) (Philips, Amsterdam, Netherlands). Nanoparticle tracking analysis (NTA) was used to analyze the size distribution of exosomes according to vesicle diameter by ZetaView (Particle Metrix, Meerbusch, Germany). A bicinchoninic acid protein assay kit was employed to determine the concentration of protein extracted from exosomes. Western blotting (WB) was used to analyze the expression of specific exosome surface markers, including CD9 and CD63.

\subsection{Experimental animals}

Male Sprague-Dawley rats (6-8 weeks old, $180-220 \mathrm{~g}, \mathrm{n}=24$ ) were obtained from Beijing Vital River Laboratory Animal Technology Co., Ltd. Rats were housed under standard laboratory conditions at a room temperature of $23^{\circ} \mathrm{C} \pm 3^{\circ} \mathrm{C}$ and a humidity of $40-65 \%$ on a 12-h light/dark cycle and were provided food and water ad libitum.

\subsection{Diabetic model establishment and intravitreal injections}

A rat model of diabetes was constructed by administering a single intraperitoneal injection of STZ (60 $\mathrm{mg} / \mathrm{kg}$ body weight) (Sigma, St. Louis, MO, USA). Nonfasting blood glucose level was measured using blood drawn from the tail vein one week after the injection of STZ. Rats with hyperglycemia (glucose levels $>16.7 \mathrm{mmol} / \mathrm{L}$ ) on three consecutive days were identified as having diabetes mellitus and selected for use in subsequent experiments. Eligible DR model rats $(n=24)$ were arbitrarily assigned to diabetic, hucMSC-Exs, and hypoxia preconditioned hucMSC-Exs (hypo-hucMSC-Exs) groups ( $\mathrm{n}=8$ rats per group). In addition, 8 healthy age-matched rats were selected as the normal control group and fed a standard diet. Intravitreal injections were administered 60 days after diabetic model establishment. All injections were administered into the right pars plana under isoflurane (2-3\% in oxygen) anesthesia. After instilling $5 \%$ betadine solution into the fornix, a sterile $33-\mathrm{G}$ needle attached to a Hamilton syringe was used to deliver $1 \mu \mathrm{L}$ solution per eye of either hypo-hucMSC-Exs, hucMSC-Exs, or PBS. Hypo-hucMSC-Exs group rats received intravitreal injection of hypo-hucMSC-Exs, the hucMSC-Exs group rats received intravitreal injection of hucMSC-Exs, and rats in the normal control and diabetic groups were administered the same volume of PBS.

\subsection{Fundus fluorescein angiography (FFA)}

FFA was performed to assess potential changes in the retinal vasculature in vivo 4, 8, and 12 weeks after intravitreal injection. Fluorescein sodium $(10 \%, 0.2 \mathrm{ml}$ ) (Akorn Inc., Lake Forest, IL, USA) was administered via intravenous injection. Angiography was performed (Heidelberg HRT2, Heidelberg, Germany) to investigate for signs of vascular leakage or changes in vessel tortuosity.

\subsection{Statistical analyses}


SPSS 21.0 (IBM Corp, Armonk, NY, USA) was used for data analysis. Data are presented as the mean \pm standard deviation. Comparisons among multiple groups were performed using one-way analysis of variance (ANOVA). Following one-way ANOVA, pairwise comparisons were conducted using the least significant difference method. $P<0.05$ was considered statistically significant.

\section{Results}

\subsection{Characterization of hucMSC-Exs and hypo-hucMSC-Exs}

We first isolated exosomes from the culture supernatants of MSCs grown in normoxic or hypoxic environments. Exosomes were then analyzed using TEM, NTA, and WB. TEM revealed nanoparticles with a typical spheroid morphology, ranging in size from 50 to $150 \mathrm{~nm}$ in diameter (Figure 1). There were no apparent differences in morphological parameters (size, shape, and electron density) between hucMSCExs and hypo-hucMSC-Exs. NTA was used to quantitatively assess the size and concentration of exosomes, which exhibited a similar size distribution, with means of 124.8 and $121.6 \mathrm{~nm}$ for hucMSC-Exs and hypo-hucMSC-Exs, respectively. Hypoxic preconditioning led to generation of more hucMSC-Exs than normoxic conditions. WB confirmed the expression of characteristic hucMSC-Exs surface markers including CD9 and CD63. Notably, the protein concentration in hypo-hucMSC-Exs was significantly higher than that in hucMSC-Exs. Hence, relative to the normoxic conditions, hucMSC-Exs were produced at higher levels following MSC exposure to hypoxic conditions.

\subsection{Intravitreal injection of hucMSC-Exs did not alter blood glucose levels}

We established a rat model of diabetes using a single intraperitoneal injection of STZ. Body weight and blood glucose levels were monitored routinely. There were no significant differences in blood glucose or body weight among all groups at baseline. At 72 hours after STZ injection, blood glucose levels of the treated rats increased significantly, and levels in selected rats were all $>16.7 \mathrm{mmol} / \mathrm{L}$. Blood glucose levels were markedly increased in the diabetic, hucMSC-Exs, and hypo-hucMSC-Exs groups compared with the normal control group. Intravitreal injection of hucMSC-Exs and hypo-hucMSC-Exs did not influence blood glucose levels, and there was no significant difference in blood glucose levels among the diabetic, hucMSC-Exs, and hypo-hucMSC-Exs groups at 4, 8, and 12 weeks after intravitreal injection ( $P>$ 0.05) (Table 1). 
Table 1

Comparison of blood glucose levels and body weight among groups

at 4 weeks after intravitreal injection

\begin{tabular}{|lll|}
\hline Group & Body weight $\mathbf{( g )}$ & Blood glucose $(\mathrm{mmol} / \mathrm{L})$ \\
\hline Control & $198.90 \pm 7.76$ & $6.10 \pm 0.52$ \\
\hline Diabetic & $177.03 \pm 8.70 *$ & $22.18 \pm 1.66 *$ \\
\hline HucMSC-Ex & $180.63 \pm 11.12 *$ & $21.40 \pm 1.21 *$ \\
\hline Hypo-hucMSC-Ex & $176.42 \pm 9.31 *$ & $21.93 \pm 1.54 *$ \\
\hline
\end{tabular}

$* P<0.05$ vs. the control group. $\mathrm{n}=8$ rats per group. Data are presented as the mean \pm standard deviation.

\subsection{Effect of hypo-hucMSC-Exs on retinal vascular abnormalities}

We performed FFA at 4, 8, and 12 weeks after intravitreal injection to assess potential changes in retinal microvasculature and permeability in vivo. The normal control group had even caliber vessels radiating from a well-defined optic nerve head. No significant changes in vascular permeability or vessel tortuosity were detected over the course of the experiment in the control group. In contrast, FFA revealed intensely hyperfluorescent lesions associated with DR. DR control group rats had tortuous vessels and massive microaneurysms in the central and peripheral retina 4 weeks after PBS administration. Representative images showing pathological features associated with DR, including microaneurysm, venous abnormalities, hemorrhage and vessel leakage, are summarized in Figure 2. Fluorescein leakage was visualized as indistinct vascular borders progressing to diffusely hazy fluorescence. Hence, longitudinal FFA revealed that rats in the DR group had extensive vascular changes manifested as heavy fluorescein leakage that showed characteristic changes over time.

As shown in Figure 3, DR control group rats had the highest increase in fluorescein leakage among the three groups of STZ-induced DR rats compared with the normal group at 12 weeks after treatment. DR group rats also presented with extensive areas of capillary non-perfusion and preretinal hemorrhage. Changes in the hucMSC-Exs group remained relatively stable, with some increases in the size and numbers of hyperfluorescent spots on follow-up examination, indicating that administration of hucMSCExs significantly reduced retinal vascular leakage. At 4 weeks post-administration, rats in the hucMSC-Exs group showed few microaneurysms, focally dilated vessels, and isolated leaky lesions. At 8 weeks after administration, vascular leakage remained relatively stable, although slight progression was observed in individual lesions. Most retinal capillaries were relatively well preserved, with only a few microaneurysms. At 12 weeks after administration, there were increased numbers of readily identifiable and isolated leaky lesions in hucMSC-Exs group rats, with retention of large areas of apparently normal retinal capillaries. There were no notable alterations 4 weeks after administration in hypo-hucMSC-Exs group rats, including no vascular leakage, venous dilatation, or tortuosity. Eight weeks post-administration, retinal vessels 
showed regular course and caliber, without microvascular changes or vessel leakage; however, at 12 weeks post-administration, there was an increase in vascular tortuosity, with evidence of some microvascular changes (e.g., microaneurysms).

The FFA data indicate that intravitreal injection of hucMSC-Exs and hypo-hucMSC-Exs decreased retinal damage. Leakage from the fluorescein spot and vascular tortuosity were less extensive in the hypohucMSC-Exs group than in the hucMSC-Exs group, indicating that DR progression was reduced in rats treated with hypo-hucMSC-Exs. Our findings suggest that hypo-hucMSC-Ex injection has a stronger potential to protect against DR progression than that of hucMSC-Exs.

\section{Discussion}

With the development of regenerative medicine, MSC transplantation is now considered a viable option for the treatment of a variety of refractory clinical diseases. MSCs have great therapeutic potential as they can self-replicate, differentiate, and regulate hematopoietic and immune cells $[14,15]$. There is evidence that MSCs act via a paracrine mechanism in the treatment of various diseases, and exosomes are important in this process $[16,17]$. The characteristics of exosomes and their application in a variety of disease models have been widely recognized by researchers. Further, technologies for exosome extraction, gene modification induction, and transplantation are under continued development. Compared with traditional MSC transplantation therapy, cell-free therapy based on MSC-Exs transplantation has clear advantages but also some disadvantages. There remain many deficiencies in treatment using MSCEx transplantation $[18,19]$. (1) Transplanted MSC-Exs cannot self-proliferate or differentiate, so the effective duration and degree of efficacy after transplantation are dose dependent, and targeting ability is weak when the transplantation dose is insufficient. (2) The steps involved in separation and extraction of high-purity MSC-Exs are complex, and the exosomes extracted in each round are far from sufficient to meet the requirements for an experiment.

Our TEM and NTA data indicated that there were no morphological differences between hucMSC-Exs and hypo-hucMSC-Exs in terms of size, shape, and electron density; however, further experiments demonstrated that hypoxic conditions promoted exosome release from MSCs.

After STZ injection, blood glucose levels were significantly higher in the DR, hucMSC-Exs, and hypohucMSC-Exs groups than those in the normal control group, while body weight was significantly lower in the DR, hucMSC-Exs, and hypo-hucMSC-Exs groups than normal controls. The differences in blood sugar and body weight among the DR, hucMSC-Exs, and hypo-hucMSC-Exs groups were not statistically significant. These findings reflect that the number of exosomes delivered by intravitreal injection of hucMSC-Exs and hypo-hucMSC-Exs is small, and the number that can reach systemic circulation through the intraocular barrier is even smaller and insufficient to lower blood sugar.

We conducted a series of in vivo FFA assessments to test our hypothesis that exosomes from cells subject to hypoxia would be superior for treatment of DE. Intravitreal injection of hucMSC-Exs and hypohucMSC-Exs were used to treat DR model rats, and our results demonstrate that both hucMSC-Exs and 
hypo-hucMSC-Exs protected against retinal vascular in this context, and that these beneficial therapeutic effects were more apparent after treatment with hypo-hucMSC-Exs.

DR is a common microvascular complication of diabetes mellitus. Exosomes can play a pathogenic role in DR occurrence and development by promoting endothelial proliferation and angiogenesis.

Monocyte-/platelet-derived exosomes are positively correlated with DR progression, particularly in patients with retinal vascular occlusion [20]. Exosomes can stimulate the coagulation cascade in retinal blood vessels, thereby aggravating microvascular damage. In addition, vitreous exosomes can accelerate proliferative DR progression by stimulating endothelial cell proliferation and promoting angiogenesis. Exosomes secreted by different tissues and cells have various effects on angiogenesis, and exosomes play a key role in the balance between angiogenesis stimulation and inhibition. Intravitreal injection of hucMSC-Exs in rats can effectively reduce the expression of inflammatory markers and adhesion molecules, thereby reducing the inflammatory response in DR [21]. Similar to our findings, Safwat et al. injected fat-derived MSC-Exs into STZ-induced diabetic rabbits via different routes (intravenous, subconjunctival, and intravitreal), and the results showed that both subconjunctival and intravitreal injection of adipose MSC-derived exosomes could protect from retinal tissue and structure damage, confirming the role of rabbit adipose MSC exosomes in retinal repair [22].

Although the protective effect of hypo-hucMSC-Exs in DR is considered a potential therapeutic strategy, its regulation and mechanism of action are not well elucidated. It remains unknown how long exosomes can survive after administration into the vitreous space. In a glaucoma model study, exosomes were delivered by weekly and monthly intravitreal injection, and the results confirmed that weekly administration of bone marrow-derived MSC-Exs could promote retinal ganglion cell survival through microRNA-dependent mechanisms with a better protective effect than monthly injections[23]. In our study, only a single injection was administered. At 4 and 8 weeks after injection, no obvious microvascular lesions were observed in the hypo-hucMSC-Exs group. After 12 weeks of intravitreal injection, the retinas in the hypo-hucMSC-Exs group showed changes characteristic of DR. In the hucMSC-Exs group, vascular lesions remained stable at 4 and 8 weeks after intravitreal injection, with only a small number of microaneurysms, local vascular dilatation, and isolated leaky lesions. Vascular lesions became worse 12 weeks after intravitreal injection, indicating that the protective effect of hypo-hucMSC-Exs on vascular injury was significantly reduced 2 months after intravitreal injection. Mead et al. used a glaucoma model to demonstrate that the neuroprotective effects of bone marrow-derived MSC-Exs were completely absent without a second injection after 1 month [23]. Whether repeated administration can enhance the protective effect of exosome hypo-hucMSC-Exs against microvascular injury in DR is unclear.

In conclusion, we established an STZ-induced DR model and used FFA to analyze the protective effects of hypo-hucMSC-Exs and hucMSC-Exs against microvascular injury. Our results suggest that hypoxia preconditioning was effective in improving the effect of hucMSC-Exs for the treatment of DR.

\section{Abbreviations}


ANOVA, analysis of variance; DR, diabetic retinopathy; FBS, fetal bovine serum; FFA, fundus fluorescein angiography; HucMSC-Exs, human umbilical cord mesenchymal stem cell exosomes; hypo-hucMSC-Exs, hypoxia-induced human umbilical cord mesenchymal stem cell exosomes; MSCs, mesenchymal stem cells MSCs, NTA, nanoparticle tracking analysis; STZ, streptozotocin; TEM, transmission electron microscope; WB, western blotting

\section{Declarations}

\section{Ethical Approval}

All experiments were conducted in accordance with the animal protocols and guidelines established by the Medicine Ethics Review Committee for Animal Experiments of our hospital and carried out in accordance with the Declaration of Helsinki.

\section{Acknowledgements}

Not applicable

\section{Consent to Publish}

All authors consent to publish

\section{Authors Contributions}

FY performed experiments, analyzed data, and wrote the manuscript; GZH performed experiments and analyzed data; WXY conducted FFA; YN conducted FFA. All authors have read and approved the manuscript.

\section{Funding}

This study was supported by the S\&T Program of Hebei (No. H2021104002). The funding source was not involved in any aspect of the study design, the collection or interpretation of data, writing of the manuscript, or the decision to publish.

\section{Competing Interests}

The authors declare that they have no competing interests to declare.

\section{Availability of data and materials}

The datasets generated for this study are available on request to the corresponding author.

\section{References}

[1] W. Wang, A.C.Y. Lo, Diabetic Retinopathy: Pathophysiology and Treatments, Int J Mol Sci, 19 (2018). 
[2] G. Mannino, C. Russo, A. Longo, C.D. Anfuso, G. Lupo, D. Lo Furno, R. Giuffrida, G. Giurdanella, Potential therapeutic applications of mesenchymal stem cells for the treatment of eye diseases, World $\mathrm{J}$ Stem Cells, 13 (2021) 632-644.

[3] X. Hu, R. Wu, L.A. Shehadeh, Q. Zhou, C. Jiang, X. Huang, L. Zhang, F. Gao, X. Liu, H. Yu, K.A. Webster, J. Wang, Severe hypoxia exerts parallel and cell-specific regulation of gene expression and alternative splicing in human mesenchymal stem cells, BMC Genomics, 15 (2014) 303.

[4] P. Page, J. DeJong, A. Bandstra, R.A. Boomsma, Effect of serum and oxygen concentration on gene expression and secretion of paracrine factors by mesenchymal stem cells, Int J Cell Biol, 2014 (2014) 601063.

[5] X. Hu, Y. Xu, Z. Zhong, Y. Wu, J. Zhao, Y. Wang, H. Cheng, M. Kong, F. Zhang, Q. Chen, J. Sun, Q. Li, J. Jin, Q. Li, L. Chen, C. Wang, H. Zhan, Y. Fan, Q. Yang, L. Yu, R. Wu, J. Liang, J. Zhu, Y. Wang, Y. Jin, Y. Lin, F. Yang, L. Jia, W. Zhu, J. Chen, H. Yu, J. Zhang, J. Wang, A Large-Scale Investigation of HypoxiaPreconditioned Allogeneic Mesenchymal Stem Cells for Myocardial Repair in Nonhuman Primates: Paracrine Activity Without Remuscularization, Circ Res, 118 (2016) 970-983.

[6] J.D. Anderson, H.J. Johansson, C.S. Graham, M. Vesterlund, M.T. Pham, C.S. Bramlett, E.N. Montgomery, M.S. Mellema, R.L. Bardini, Z. Contreras, M. Hoon, G. Bauer, K.D. Fink, B. Fury, K.J. Hendrix, F. Chedin, S. El-Andaloussi, B. Hwang, M.S. Mulligan, J. Lehtio, J.A. Nolta, Comprehensive Proteomic Analysis of Mesenchymal Stem Cell Exosomes Reveals Modulation of Angiogenesis via Nuclear FactorKappaB Signaling, Stem Cells, 34 (2016) 601-613.

[7] J. Zhu, K. Lu, N. Zhang, Y. Zhao, Q. Ma, J. Shen, Y. Lin, P. Xiang, Y. Tang, X. Hu, J. Chen, W. Zhu, K.A. Webster, J. Wang, H. Yu, Myocardial reparative functions of exosomes from mesenchymal stem cells are enhanced by hypoxia treatment of the cells via transferring microRNA-210 in an nSMase2-dependent way, Artif Cells Nanomed Biotechnol, 46 (2018) 1659-1670.

[8] B.C. Lee, K.S. Kang, Functional enhancement strategies for immunomodulation of mesenchymal stem cells and their therapeutic application, Stem Cell Res Ther, 11 (2020) 397.

[9] N. Ishiuchi, A. Nakashima, S. Doi, K. Yoshida, S. Maeda, R. Kanai, Y. Yamada, T. Ike, T. Doi, Y. Kato, T. Masaki, Hypoxia-preconditioned mesenchymal stem cells prevent renal fibrosis and inflammation in ischemia-reperfusion rats, Stem Cell Res Ther, 11 (2020) 130.

[10] N.A. Hofmann, A. Ortner, R.O. Jacamo, A. Reinisch, K. Schallmoser, R. Rohban, N. Etchart, M. Fruehwirth, C. Beham-Schmid, M. Andreeff, D. Strunk, Oxygen sensing mesenchymal progenitors promote neo-vasculogenesis in a humanized mouse model in vivo, PLoS One, 7 (2012) e44468.

[11] C.S. Zhang, K. Shao, C.W. Liu, C.J. Li, B.T. Yu, Hypoxic preconditioning BMSCs-exosomes inhibit cardiomyocyte apoptosis after acute myocardial infarction by upregulating microRNA-24, Eur Rev Med Pharmacol Sci, 23 (2019) 6691-6699. 
[12] W. Liu, Y. Rong, J. Wang, Z. Zhou, X. Ge, C. Ji, D. Jiang, F. Gong, L. Li, J. Chen, S. Zhao, F. Kong, C. Gu, J. Fan, W. Cai, Exosome-shuttled miR-216a-5p from hypoxic preconditioned mesenchymal stem cells repair traumatic spinal cord injury by shifting microglial M1/M2 polarization, J Neuroinflammation, 17 (2020) 47.

[13] W. Liu, L. Li, Y. Rong, D. Qian, J. Chen, Z. Zhou, Y. Luo, D. Jiang, L. Cheng, S. Zhao, F. Kong, J. Wang, Z. Zhou, T. Xu, F. Gong, Y. Huang, C. Gu, X. Zhao, J. Bai, F. Wang, W. Zhao, L. Zhang, X. Li, G. Yin, J. Fan, W. Cai, Hypoxic mesenchymal stem cell-derived exosomes promote bone fracture healing by the transfer of miR-126, Acta Biomater, 103 (2020) 196-212.

[14] F. Gubert, J.S. da Silva, J.F. Vasques, R.G. de Jesus Goncalves, R.S. Martins, M.P.L. de Sa, R. MendezOtero, G. Zapata-Sudo, Mesenchymal Stem Cells Therapies on Fibrotic Heart Diseases, Int J Mol Sci, 22 (2021).

[15] L.T. Wang, K.J. Liu, H.K. Sytwu, M.L. Yen, B.L. Yen, Advances in mesenchymal stem cell therapy for immune and inflammatory diseases: Use of cell-free products and human pluripotent stem cell-derived mesenchymal stem cells, Stem Cells Transl Med, (2021).

[16] T. Katsuda, N. Kosaka, F. Takeshita, T. Ochiya, The therapeutic potential of mesenchymal stem cellderived extracellular vesicles, Proteomics, 13 (2013) 1637-1653.

[17] N.P. Hessvik, A. Llorente, Current knowledge on exosome biogenesis and release, Cell Mol Life Sci, 75 (2018) 193-208.

[18] D.G. Phinney, M.F. Pittenger, Concise Review: MSC-Derived Exosomes for Cell-Free Therapy, Stem Cells, 35 (2017) 851-858.

[19] L. Otero-Ortega, F. Laso-Garcia, M. Gomez-de Frutos, B. Fuentes, L. Diekhorst, E. Diez-Tejedor, M. Gutierrez-Fernandez, Role of Exosomes as a Treatment and Potential Biomarker for Stroke, Transl Stroke Res, 10 (2019) 241-249.

[20] N. Ogata, S. Nomura, A. Shouzu, M. Imaizumi, M. Arichi, M. Matsumura, Elevation of monocytederived microparticles in patients with diabetic retinopathy, Diabetes Res Clin Pract, 73 (2006) 241-248.

[21] W. Zhang, Y. Wang, Y. Kong, Exosomes Derived From Mesenchymal Stem Cells Modulate miR-126 to Ameliorate Hyperglycemia-Induced Retinal Inflammation Via Targeting HMGB1, Invest Ophthalmol Vis Sci, 60 (2019) 294-303.

[22] A. Safwat, D. Sabry, A. Ragiae, E. Amer, R.H. Mahmoud, R.M. Shamardan, Adipose mesenchymal stem cells-derived exosomes attenuate retina degeneration of streptozotocin-induced diabetes in rabbits, J Circ Biomark, 7 (2018) 1849454418807827.

[23] B. Mead, J. Amaral, S. Tomarev, Mesenchymal Stem Cell-Derived Small Extracellular Vesicles Promote Neuroprotection in Rodent Models of Glaucoma, Invest Ophthalmol Vis Sci, 59 (2018) $702-714$. 


\section{Figures}

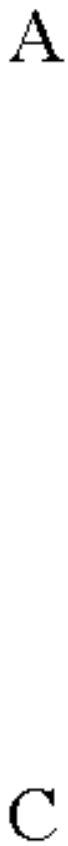

$\mathrm{B}$
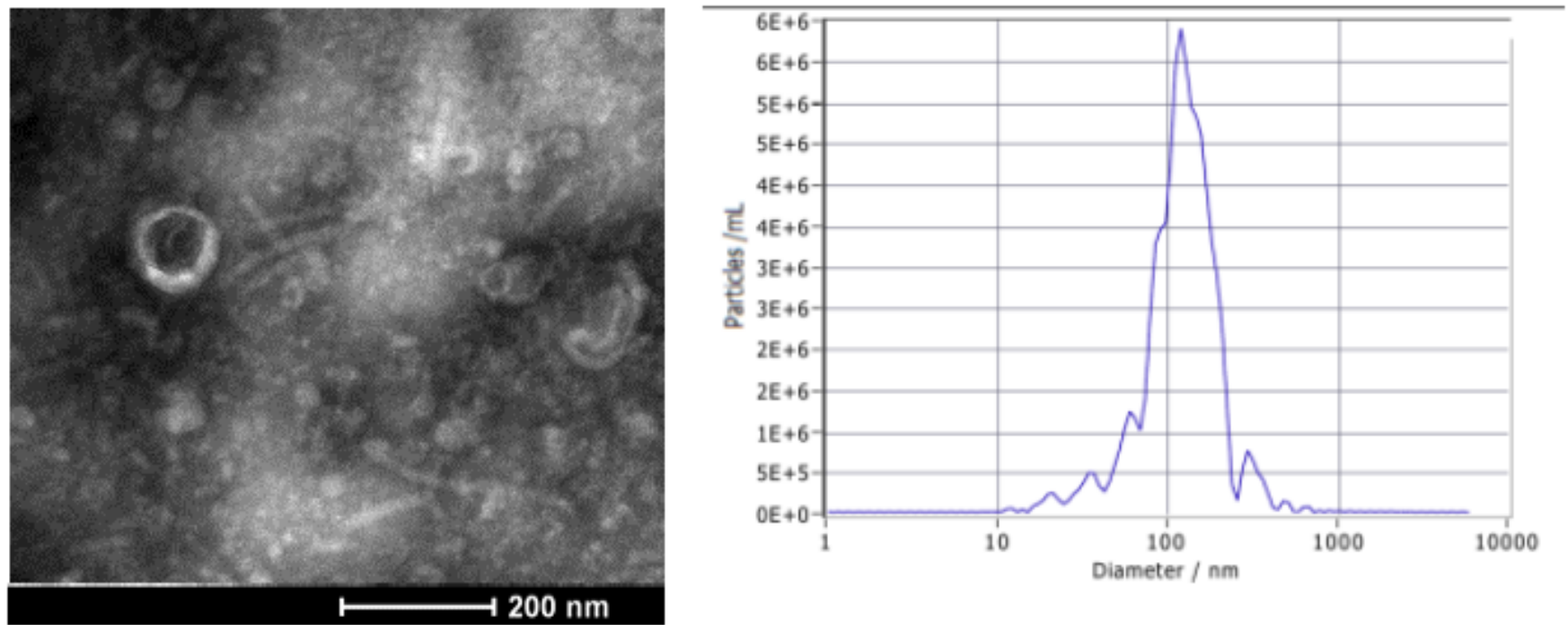

$\mathrm{C}$
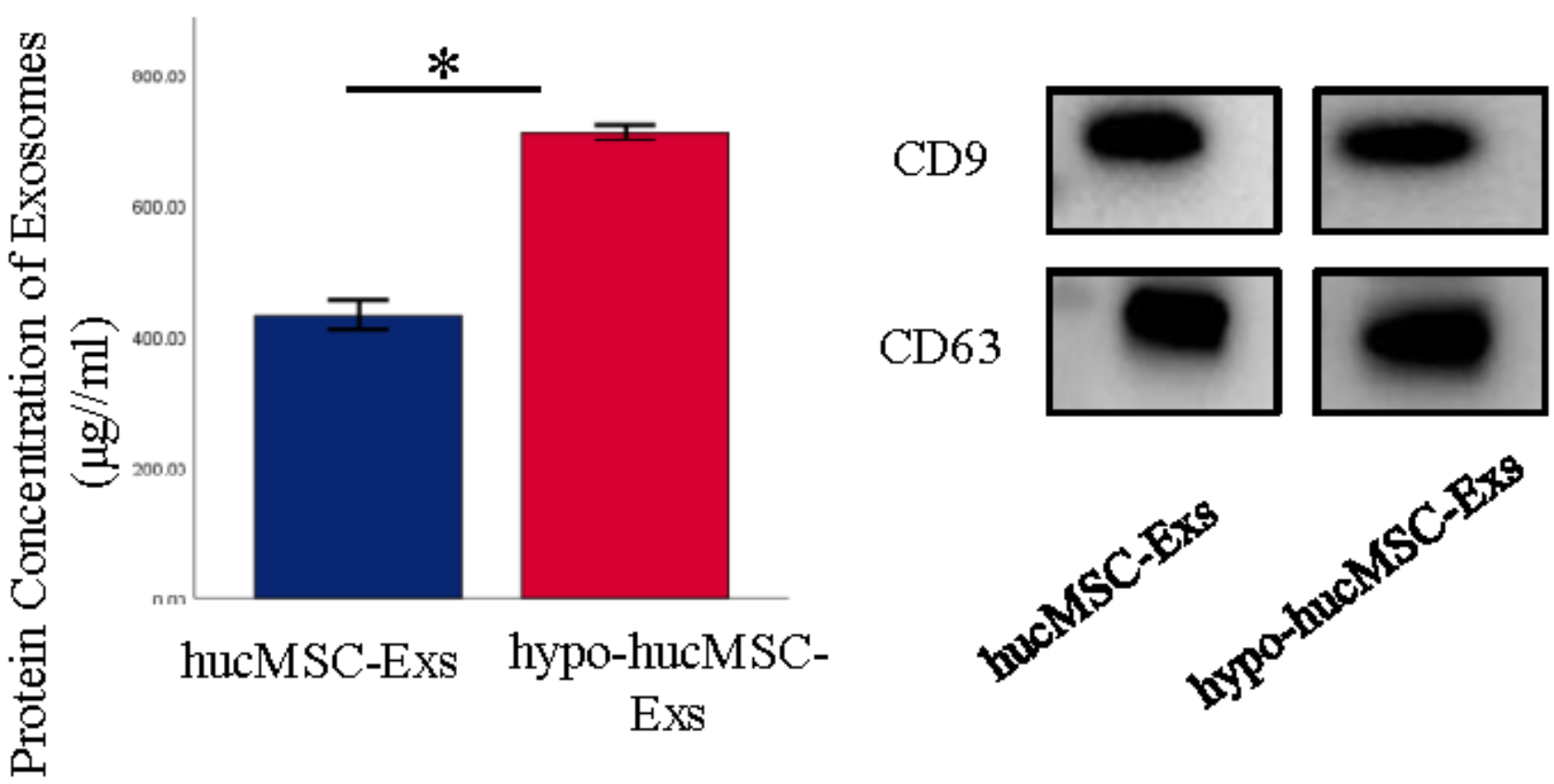

Figure 1

Characterization of exosomes. (A) Morphology of exosomes observed under transmission electron microscopy. (B) Nanoparticle tracking analysis of exosomes. (C) Protein concentrations in exosomes measured using a bicinchoninic acid protein assay kit. (D) Expression of the characteristic exosomes surface markers, CD9 and CD63, analyzed by Western blot analysis. ${ }^{*}<0.05$. 

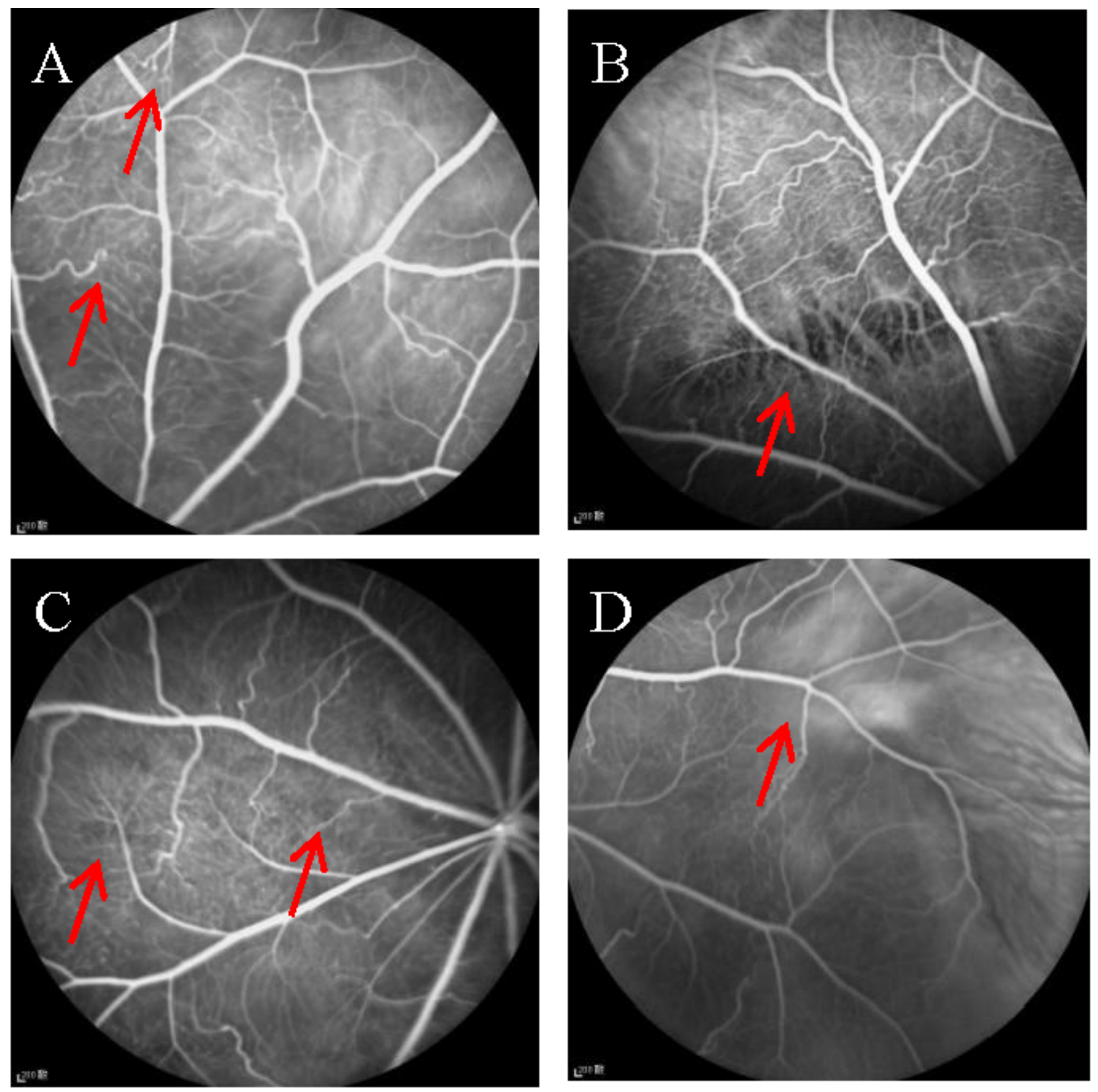

Figure 2

FFA showing different features of DR. Eyes with DR showing microaneurysms, venous dilatation, and tortuosity (A), hemorrhages (B), zones of capillary non-perfusion (C), and hyperfluorescent lesions (D). 
4 weeks

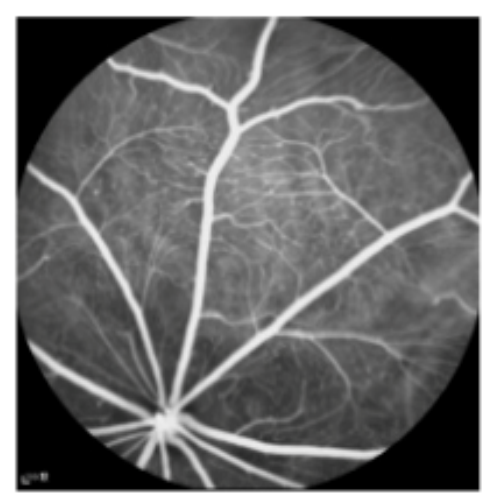

DR group
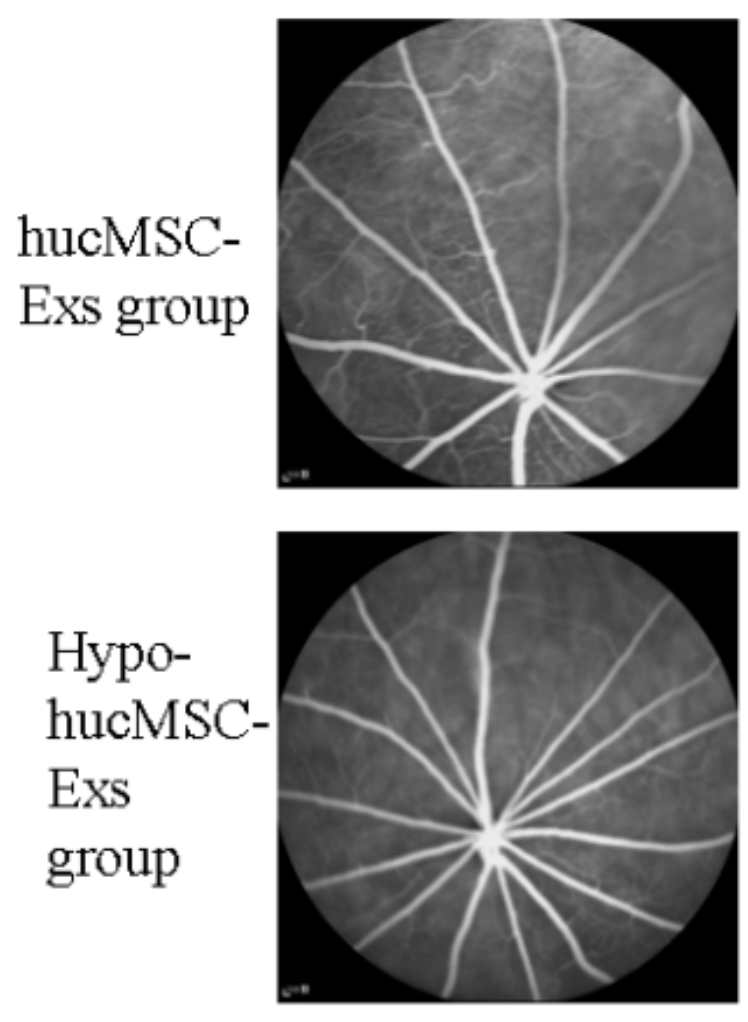

8 weeks
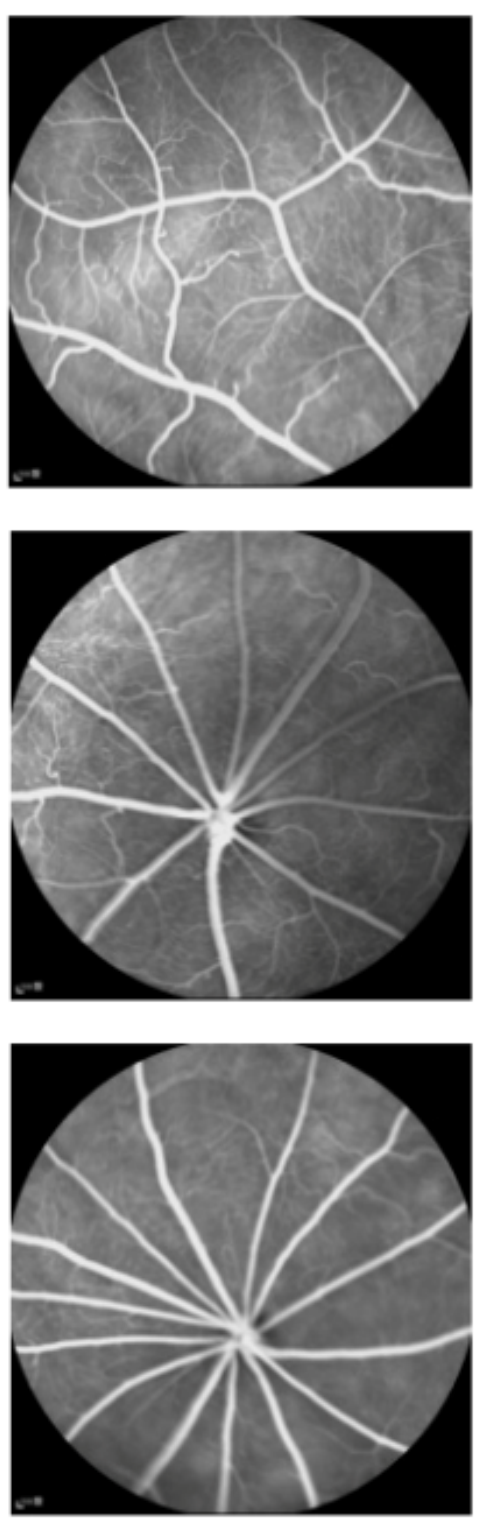
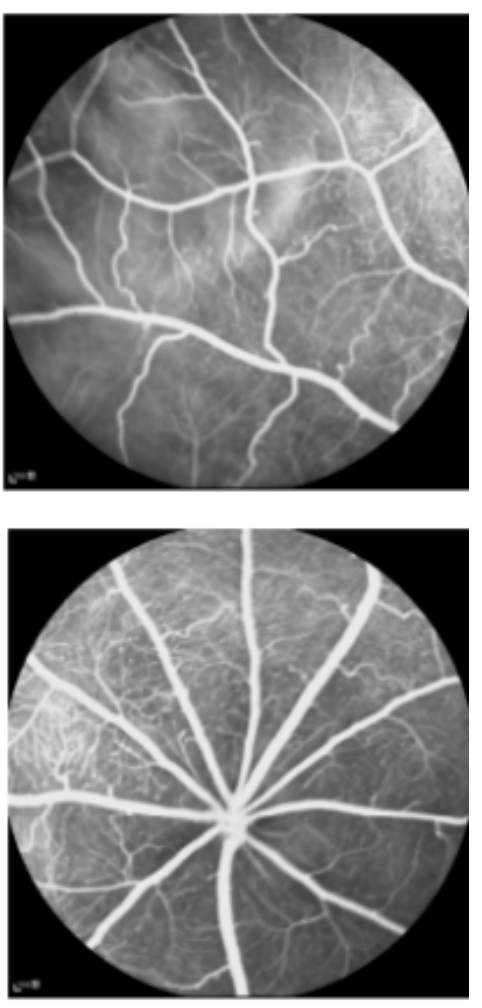

12 weeks

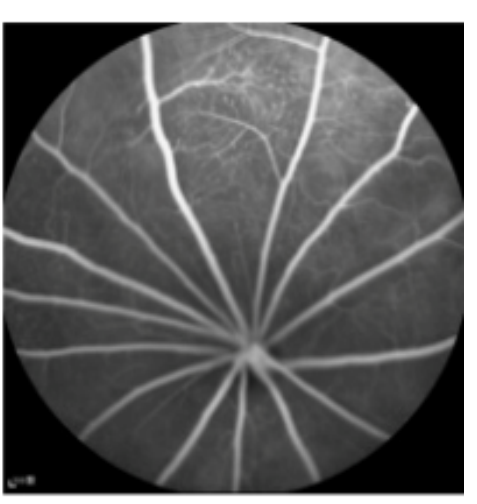

\section{Figure 3}

Representative longitudinal FFA images. Microaneurysms and vascular tortuosity were observed in DR group rats 4 weeks after administration of PBS. Increased fluorescein leakage was evident at 12 weeks. At 4 weeks after intravitreal injections, hucMSC-Exs group rats showed fewer microaneurysms and dilated vessels. Leaky lesions and capillary non-perfusion were significantly decreased in hucMSC-Exs group rats relative to controls at 12 weeks. No alterations worthy of note in were detected in hypo- 
hucMSC-Exs group rats at 4 and 8 weeks. At 12 weeks after intravitreal injections of hypo-hucMSC-Exs, increased vascular tortuosity, with some microvascular changes, such as microaneurysms, was observed. 